\title{
Device-Free Indoor Localization for AAL Applications
}

\author{
Paolo Barsocchi ${ }^{1}$, Francesco Potorti ${ }^{1}$, and Paolo Nepa ${ }^{2 \star}$ \\ 1 ISTI-CNR, Pisa Research Area, Via G.Moruzzi 1, 56124, Pisa, Italy \\ paolo.barsocchi, Potorti@isti.cnr.it, \\ 2 Department of Information Engineering, University of Pisa, Via Caruso 16, 56122, \\ Pisa, Italy \\ paolo.nepa@iet.unipi.it
}

\begin{abstract}
We present a device-free localization method oriented to Ambient Assisted Living applications. The system exploits the received signal strength (RSS) measured by fixed wireless communications devices, whose position is known a priori, in order to localize a person in the transmission coverage area. The proposed localization system is passive, by not requiring the user to wear anything, and is able to trade complexity for accuracy by simply changing the number of deployed devices. The presentation is validated by an indoor experimental study.
\end{abstract}

Key words: RSS-based localization, AAL, tracking

\section{Introduction}

Localization and tracking of mobile users in indoor environments is one of the main components (often an enabling one) in Ambient Assisted Living (AAL) [1] applications. AAL aims at improving the quality of life of elderly or disabled people, by assisting them in their daily life, in order to preserve their autonomy and by making them feeling included, protected and secure in the places where they live or work (typically their home, their office, the hospital and any other place where they may spend a significant part of their time). These objectives can be granted only if the appropriate services are delivered to the users in the right place at the right time. Localization and tracking of objects can be achieved by means of a large number of different technologies, however only few of them are suitable for AAL applications, since they should be non-invasive on the users, they must be suited to the deployment in the user houses at a reasonable cost, and they should be accepted by the users themselves. Accuracy in the position estimation is less critical than in other applications (accuracies in the order of the centimeter or below are typically not required). As an example, the EvAAL

\footnotetext{
* This work was supported by the European Commission under the UNIVERsal open platform and reference Specification for Ambient Assisted Living IP (universAAL, FP7-247950) within the $7^{\text {th }}$ Research Framework Programme.
} 
(Evaluating AAL system through benchmark $[1,2])$ competition compares indoor localization systems for AAL applications by taking into account metrics like user acceptance, availability, interoperability, and installation complexity, in addition to accuracy. For AAL applications, the required accuracy depends on the specific application that uses the localization component. For example, if the application needs to know if the person goes to the living room to switch on the TV, the requested accuracy is in the order of meters. On the other hand, if the application controls the cookware, a more precise indoor localization system is required. In other words, a localization system that changes its accuracy in accordance with the application needs is desirable. The localization solutions proposed in the literature are mainly focused on achieving as good an accuracy as possible, without adapting to the different requirements depending on the environment, and do not generally provide a way to estimate the attainable accuracy in advance. All in all, the literature is lacking solutions attractive for AAL spaces, which localize a person with an accuracy performance defined in advance in accordance with the requirements of the applications.

Considering all the above constraints, a promising technology for these services is based on Wireless Sensor Networks (WSN) [3], due to their advantages in terms of costs and time for effective deployment. Within such WSNs, it is possible to estimate the location of a user by exploiting the Received Signal Strength (RSS) information, which is a measure of the power of a received radio signal that can be obtained from almost any wireless device. The RSS measured among fixed devices (whose position is known) and mobile devices (carried by the user) is leveraged by algorithms that estimate the coordinates of the user positions. In a smart environment, where the ambience is instrumented with sensors and wireless communication devices, the marginal cost of implementing an RSS-based localisation system can be very low, as it can leverage the existing installed hardware.

Device-free localization techniques are a subset of the above ones: they do not need a mobile device worn by the person to be localized $[4,5]$. These systems are based on large set of small wireless devices spread over the area of interest in order to create a dense mesh, and exploit the RSS observed by each device on the links connecting it to other devices. A user moving within the area modifies the RSS pattern in a way that depends on his location; radio imaging therefore exploits the RSS measurements observed along the inter-device links to obtain a reconstruction of the object trajectory. The two main drawbacks of these methods are the large number of devices that must be deployed in the environment and the incapability of discriminating among users. Association with a sufficiently smart tracking system may help with the latter problem, while a complete solution is only possible in association with other techniques (usually non device-free, like RFID). The achieved accuracy is usually around a dozen centimeters, which is more than enough for AAL applications, where $50 \mathrm{~cm}$ can be considered acceptable $[1,2]$.

In this paper we propose an indoor localization system that is able to estimate in advance the maximum error, thus matching the AAL application needs, and 
is particularly indicated for AAL environment where good user acceptance is essential. The proposed system works even with a small number of installed devices, thus eliminationg the first of the mentioned drawbacks of device-free localization techniques.

\section{Our Solution in a Nutshell}

The proposed localization system works by placing a number of sensors (hereafter called anchors) around the area of interest. Each device is capable of transmitting and receiving wireless thus creating a network of radio links that encompasses the whole area (figure 1). A user crossing the Line-of-Sight (LOS) link between two anchors causes signal fading through the link. Identifying power reduction on a given radio link can be exploited to know the area where the user is going into. As shown in figure 1, if the user enters the room and the detection algorithm detects that the LOS link between anchors 1 and 2 is affected, the localization system infers that the user is entering the subarea $A_{1}$, and will indicate the coordinates of the centroid of that subarea. The proposed localization system is strongly influenced by the detection algorithm, which will be described and evaluated in section 3.3 .

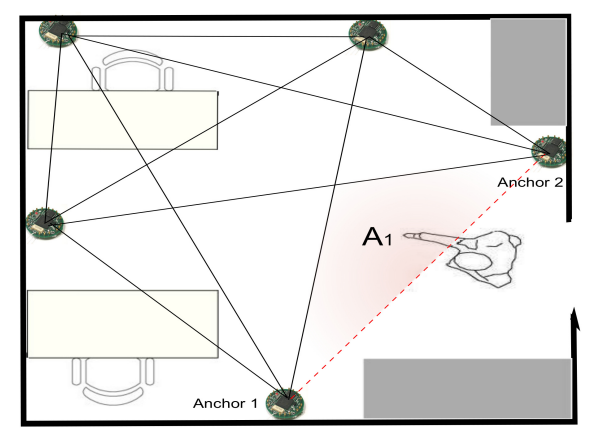

Fig. 1. The proposed AAL compliant device-free localization system.

The proposed localization system is able to:

- make a priori estimation of the maximum error by evaluating the maximum distance between the centroid and the farthest point of each subarea;

- adapt the required accuracy to the application needs at installation time. If the application needs a higher location accuracy in a given area, the anchors can be deployed in order to create smaller subareas - on the contrary, if the application needs a lower accuracy, larger subareas can be planned.

According to the metric proposed in $[1,2]$, the proposed system is particularly suitable for AAL applications, since: 
- the installation complexity can be made low by simulating the anchor deployment before deployment;

- user acceptance benefits from the users not having to carry anything on them.

\section{Experimental Evaluation}

In this section we describe the environment setup of the measurement campaign conducted at the first floor of CNR-ISTI, the detection algorithm and its performance, and comment on the proposed localization algorithm.

\subsection{Setup}

We deployed a network of five IRIS wireless sensors nodes [6] in a $23 \mathrm{~m}^{2}$ indoor laboratory, at about $120 \mathrm{~cm}$ above the floor. The nodes operate in the $2.4 \mathrm{GHz}$ frequency band using the IEEE 802.15.4 transmission protocol. We used a modified version of the management software Spin [7], which implements a tokenpassing protocol to schedule node transmission. Anchors transmit a packet in broadcast, so all other anchors receive the packet and perform the RSS measurements. Packets contain the previous RSS measurements and are collected by a base station along with the node's unique ID, for storage and later processing. The data collected during the experiment consisted of more than 40000 RSS measurements for a total time of $668 \mathrm{~s}$. The experimental setup is shown in Figure 2. Only the user to be localized was present in the area during the experiment.

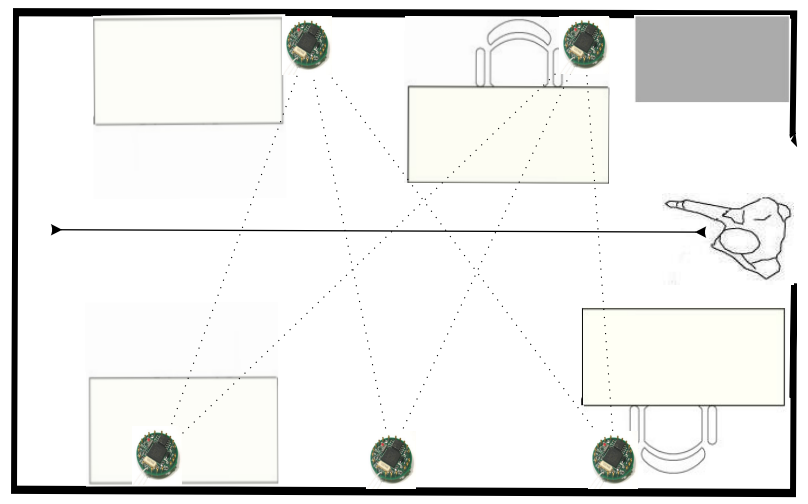

Fig. 2. Environment setup: 5 anchors placed close to the lateral walls of the room, at about $120 \mathrm{~cm}$ height. The straight line indicates the actual path followed during the experiment. 


\subsection{RSS behaviour}

In this section we show the RSS behavior during one of the data acquisition phases. Each anchor $j$ collect the experienced RSS $r_{i, j}$ from all other anchors $i \in N_{j}$, where $N_{j}$ is the set of neighbors of $j$. Figure 3 shows the RSS and the variance $\sigma_{1,4}^{2}$ experienced between anchors 1 and 4 . When the user interferes with the LOS link between the anchors 1 and 4, the RSS value decreases; when the user passes over the 1-4 radio link, the RSS value returns to normal. Estimating $\sigma_{1,4}^{2}$ is a reliable way of detecting the crossing event. As illustrated in figure 3, the temporal variance of the RSS values experiences 60 peaks, as expected since during the experiment the user crossed the link 60 times.

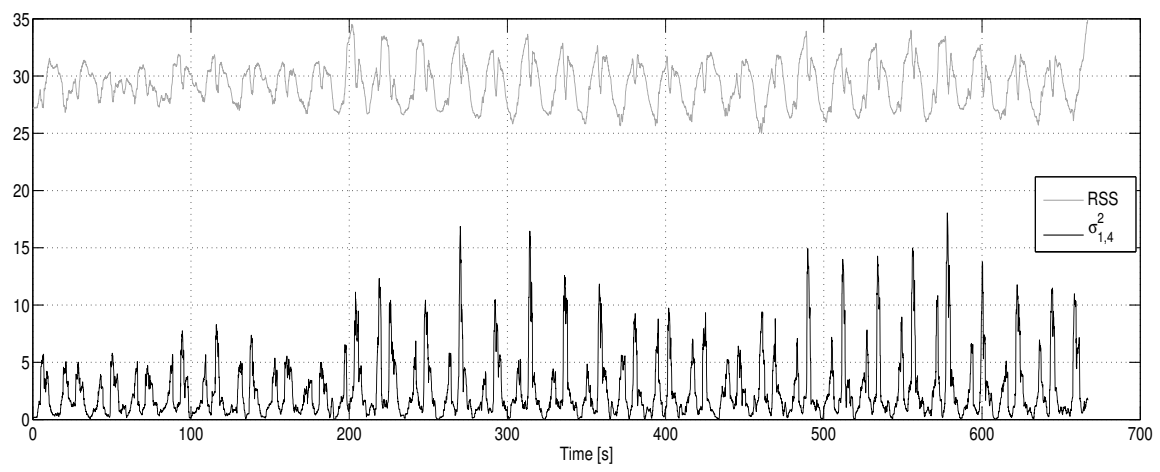

Fig. 3. RSS values and variance of the link between anchors 1 and 4 .

\subsection{Detection algorithm}

The proposed detection algorithm is based on the observation of the RSS characteristics highlighted in previous section. As shown in related papers [8], the RSS variance is a valuable parameter to detect events in wireless systems. When the RSS variance increases it means that something happens that changes the RSS behavior for a while. In this particular case, the variance of the RSS increases when a person crosses the LOS link between a couple of anchors. When this event ends, the RSS variance goes back to its unperturbed value. Starting from such considerations we deployed an algorithm as follows:

1. given a receiver $j$ and a transmitter $i \in N_{j}$ (where $N_{j}$ is the set of neighbors of $j$ ) a number $W$ of RSS measurements is collected $R_{i, j}=\left\{r_{i, j}^{(w)}\right\}_{w=1}^{w=W}$

2. the RSS variance $\sigma_{R_{i, j}}^{2}$ of the $W$ measures is evaluated;

3. when $\sigma_{R_{i, j}}^{2}<=\gamma$, the link between $i$ and $j$ is assumed to be unaffected and we go back to step 1 for the first measurement of the next window;

4. when $\sigma_{R_{i, j}}^{2}>\gamma$, user crossing is detected and we go back to step 1 for the first measurement of the next window. 
Performance The detection test can be viewed as a choice between two events $H_{0}$ and $H_{1}$.

$$
\begin{aligned}
& H_{0}: \sigma_{R_{i, j}} \leq \gamma \\
& H_{1}: \sigma_{R_{i, j}}>\gamma
\end{aligned}
$$

Since the variances $\sigma_{R_{i, j}}^{2}$ are random variables, their conditional density functions are denoted as $f_{\sigma_{i, j}}\left(\sigma \mid H_{0}\right)$ and $f_{\sigma_{i, j}}\left(\sigma \mid H_{1}\right)$. The performance of a detector will be given by using the probability of false alarm $P_{F A}$ and probability of detection $P_{D}$ defined, on a $1 \mathrm{~s}$ window time, as:

$$
\begin{aligned}
& P_{F A}=\int_{x=\gamma}^{\infty} f_{\sigma_{i, j}}\left(x \mid H_{0}\right) \mathrm{d} x \\
& P_{D}=\int_{x=\gamma}^{\infty} f_{\sigma_{i, j}}\left(x \mid H_{1}\right) \mathrm{d} x
\end{aligned}
$$

The probability of missed detection $P_{M}$, is $P_{M}=1-P_{D}$. Since these probabilities are a function of $\gamma$, we can trade lower false alarm for missed detection. The objective of the experimental activity is to evaluate the above trade-off and show the achievable performance. To this purpose the receiver operating characteristic (ROC) curve is computed. The ROC curve is a classical method for displaying the performance of a detection algorithm by plotting the probability of false alarm $P_{F A}$ versus probability of detection $P_{D}$ (hit rate). The threshold $\gamma$ is not shown explicitly, but for a particular value of $\gamma$, the detector would achieve specific $P_{F A}(\gamma)$ and $P_{D}(\gamma)$ values. We tested a range of $\gamma$ values and plotted $P_{D}(\gamma)$ versus $P_{F A}(\gamma)$ in a single ROC curve.

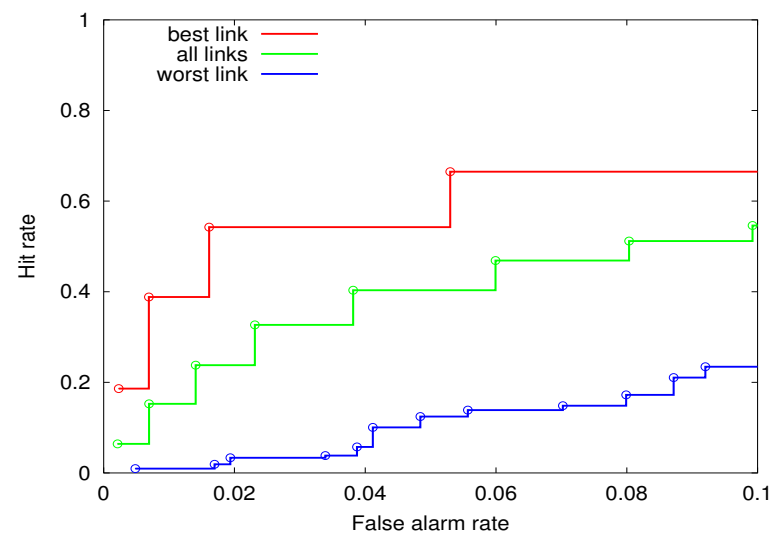

Fig. 4. ROC curve of the proposed detection algorithm. Window length is $1 \mathrm{~s}$.

The quality of the links is higly variable, as shown by the distance between the curves relative to the worst and the best link. Also, the performance of even the best link is not very good. It will be interesting to note that the localization performance is good even in the presence of a rather poor and highly variable ROC performance. 
The values of $\gamma$ relative to the points on the curve are roughly inversely proportional to the false alarm rate with a value around $\operatorname{range}\left(r_{i, j}\right) / 20$ for a $10 \%$ false alarm.

\subsection{Localization error}

We define the performance of the localization algorithm in terms of error, that is the distance between the estimated and the actual position.

It may happen that two or more events (LOS link obstruction) are detected simultaneously, either because two rays are obstructed or because of false alarms: in this cas the localization algorithm estimate is the centroid of the positions indicated by all the events. In this experiment we set the optimal detection algorithm parameters as described in section 3.3. Figure 5 shows the CDF obtained by using the proposed localization algorithm (solid line) compared to the one that the algorithm would obtain for a $100 \%$ detection probability and a null false alarm probability (dotted line). In our experiment, the localization algorithm exhibited a median error of $1 \mathrm{~m}$, with an error less than $2 \mathrm{~m}$ in $80 \%$ of cases; the theoretical median error is about $0.4 \mathrm{~m}$ and in $80 \%$ of cases the error is below $1 \mathrm{~m}$. Root mean square error is $1.7 \mathrm{~m}$ for the real case, $0.8 \mathrm{~m}$ for the theoretical case. We can decrease the theoretical error at will by increasing the number of deployed anchors.

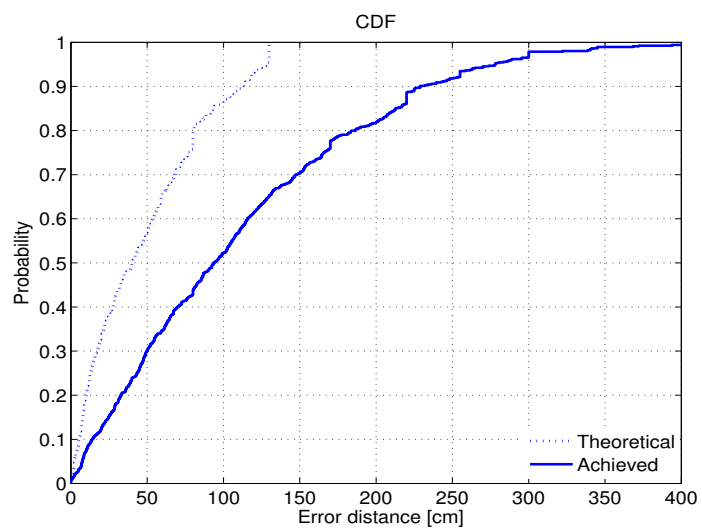

Fig. 5. Cumulative distribution function of the error measured during the experiment depicted in 3. The theoretical case assumes perfect detection.

\section{Conclusion}

We have experimentally observed that, with as few as 5 anchors in a room of about $23 \mathrm{~m}^{2}$, our method achieves a median error of $1 \mathrm{~m}$, with an RMS of $1.7 \mathrm{~m}$, 
and an attainable median error of $0.4 \mathrm{~m}$, with an RMS of $0.8 \mathrm{~m}$. Work is in progress to improve the performance of the detection algorithm, to minimize the probability of false alarm and to increase the detection probability.

\section{References}

1. Barsocchi, P., Potortì, F., Gil, A.M.M.: Comparing aal indoor localization systems. In: Springer's Communications in Computer and Information Science (CCIS) series. (2012)

2. Salvi, D., Barsocchi, P., Arredondo, M.T., Ramos, J.P.L.: Evaal, evaluating aal systems through competitive benchmarking, the experience of the 1st competition. In: Springer's Communications in Computer and Information Science (CCIS) series. (2012)

3. Barsocchi, P., Lenzi, S., Chessa, S., Furfari, F.: Automatic virtual calibration of range-based indoor localization systems. Wireless Communications and Mobile Computing (2011)

4. Savazzi, S., Nicoli, M., Riva, M.: Radio imaging by cooperative wireless network: Localization algorithms and experiments. In: WCNC. (2012) 2357-2361

5. Wilson, J., Patwari, N.: Radio tomographic imaging with wireless networks. IEEE Trans. Mob. Comput. 9(5) (2010) 621-632

6. Crossbow Technology Inc.: Iris crossbow data sheet

7. Wilson, J., Patwari, N.: Spin: A token ring protocol for RSS collection

8. Wilson, J., Patwari, N.: See-through walls: Motion tracking using variance-based radio tomography networks. IEEE Trans. Mob. Comput. 10(5) (2011) 612-621 